\title{
Exercice d'analyse de paysage dans la région Martigny-Chamoson
}

Fig. 1: Situation générale

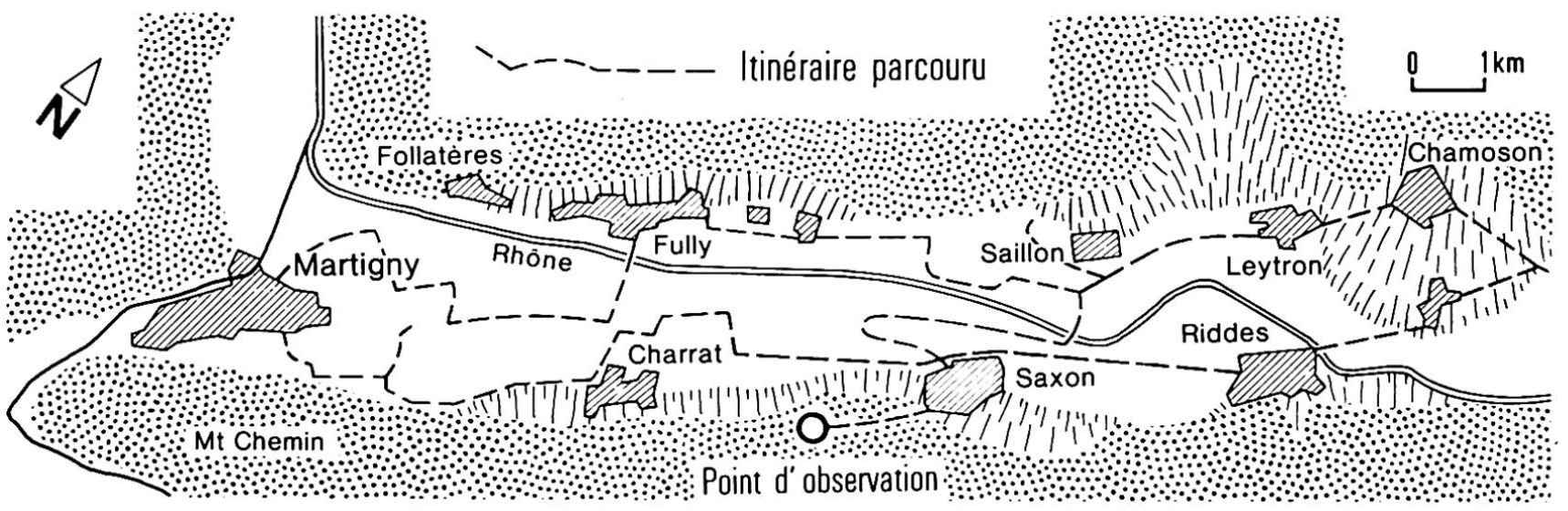

Travail réalisé après observation à partir du point 578.800/109.300 et un parcours a travers la région

\section{Analyse du paysage physique et végétal}

\section{Les grandes divisions du paysage}

Vu du point 568.800/109. 300 dominant Saxon, le paysage offre trois grands ensembles:la plaine, le coteau nord et le coteau sud. Au-dela du premier plan, on distingue en outre la cluse du Rhône et le plateau d'Ovronnaz.

\section{La plaine}

Elle semble s'arreter brusquement à la hauteur du coude de Martigny. Caractérisée par sa pente très faible et sa grande platitude, elle s'appuie à l'est et à l'ouest sur de grands cônes de déjection, qui rompent la monotonie topographique du fond de vallée. De notre point de vue, le cône de la Drance est peu visible; il n'offre en effet qu'une dénivellation très faible. En revanche, il s'étend largement et bouscule le Rhône contre le pied de l'arête des Follatères, entravant l'écoulement du fleuve et provoquant la convergence des différents canaux d'assainissement vers le point de confluence de la Drance et du Rhône. La large cuvette peu profonde du Guercey s'étale a l'amont de ces alluvions récentes. Dans l'atlas topographique de 1915 , elle se présente comme un vaste marécage. Interrompue par l'extension vers la plaine du village de Charrat, la dépression - un peu rétrécie - se prolonge ensuite jusqu'a Saxon. De notre belvédère, nous pouvons repérer - à l'ouest de ce dernier village - sur quelques centaines de metres, l'ancien lit du Petit Rhône, bordé d'abondantes roselieres. Cette partie de la plaine longeant le coteau sud est aujourd'hui assainie. Sa mise en valeur récente se devine à la faible densité du verger; les jardins fruitiers, rares encore, sont peu touffus et laissent une large part aux terres labourées. Le reste de l'espace Martigny/Riddes se cache sous un dense manteau d'arbres fruitiers, ombrageant les cultures marafchères. Notre parcours a travers champs nous a permis, en outre, d'observer la nature changeante du sol; constitue dans l'ensemble de limons argileux et fertiles, ici ou là, en l'espace de peu de metres, sa couleur s'éclaircit;il n'est plus formé que de sables ou de graviers délavés, dans lesquels végètent des poiriers et des pommiers rabougris, alors que ces memes essences prosperent dans les sols fertiles voisins.

La transition entre la plaine et le coteau est brutale de Saillon a Fully, ainsi qu'aux environs du Guercey. Ailleurs, des séries de cônes de déjection contigus, mêlés à des éboulis, festonnent le pied des versants et atté -

Dr. M. Roten, 43 Rte. de Loèche, 1950 Sion 
Fig. 2: La région Martigny-Charrat d'après At. Siegfr. 1915

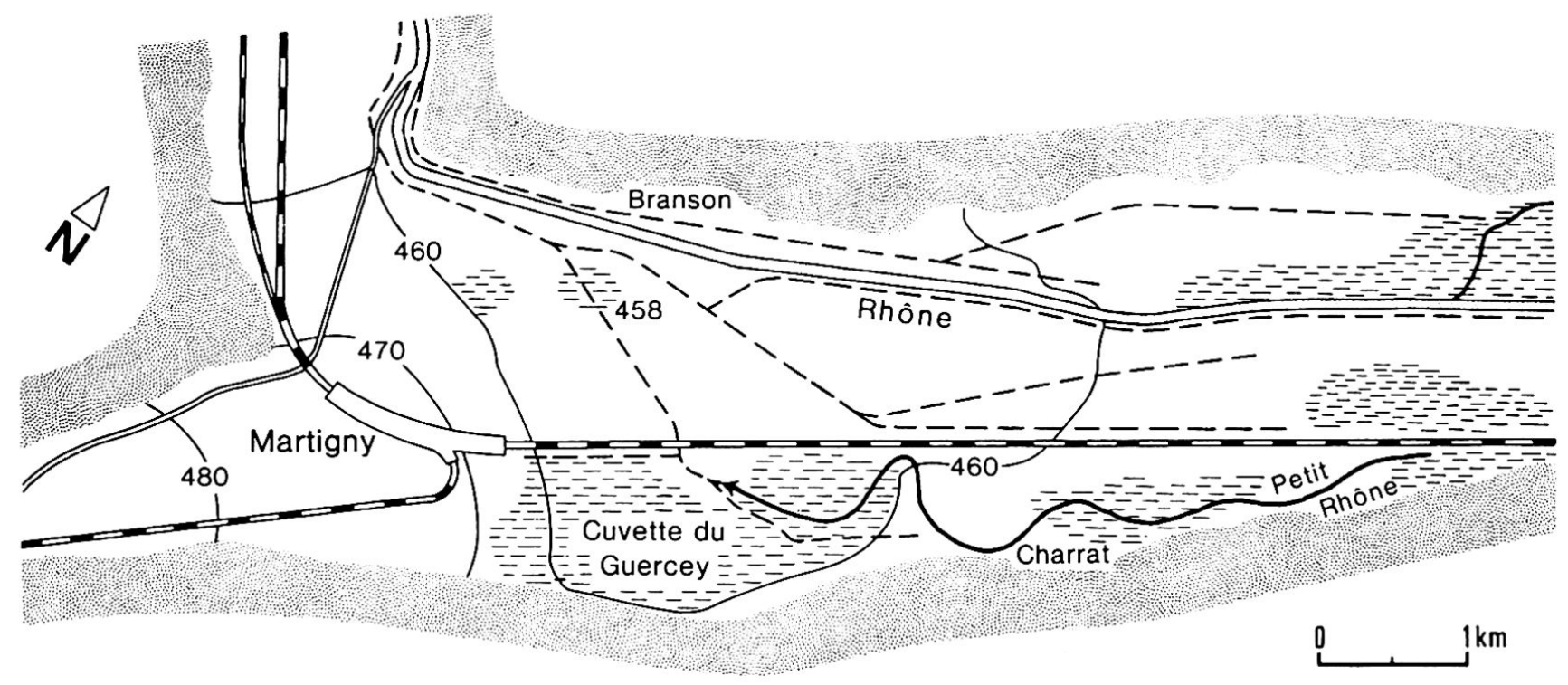

nuent les contrastes topographiques. Ainsi en est-il à l'ouest de Charrat où ils sont si rapprochésqu'ils se chevauchent partiellement. L'absence de ruisseau et même de tout lit d'écoulement à leur amont, ainsi que la nature en majorité morainique de leur matériel, permettent de supposer qu'ils découlent, au moins partiellement, d'une genèse de glissement. Dans la région de Fully, pas moins de sept cônes habillent le bas des versants; deux d'entre eux (ceux de Fully et de Mazembroz) sont largement recouverts d'éboulis; épierrés systématiquement, ils sont aujourd'hui quadrillés par d'imposants talus de cailloux: "les murgères". A partir de Saillon, la transition entre le versant nord et le fond de la vallée est toute différente; la pente se fait plus douce. Les petites terrasses de Saillon, le cône de déjection de la Salentze et le coteau fluent de Leytron constituent la zone de contact.

Plus loin, le vaste cône de Chamoson s'élève progressivement jusqu'à plus de 800 metres; il permet, à la hauteur de Grugnay, de confondre le coteau et la plaine. Large de 4 a $5 \mathrm{~km}$, il s'incline sur $3 \mathrm{~km}$ en direction du village de Riddes assis sur les alluvions moins abon- dantes de la Faraz. Ces deux cônes de déjection tracent une limite géographique entre le Valais central et le Bas-Valais-reconnue par la constitution cantonale comme frontiere politique. L'encombrement crée par cette accumulation d'alluvions a peut-être, autrefois, ralenti l'écoulement des eaux des cuvettes d'Ardon et de Vétroz, et favorisé la persis tance de marécages. Aujourd'hui, le Rhône s'est légèrement encaissé entre les deux

Fig. 3: Saxon (Photo N.F.)

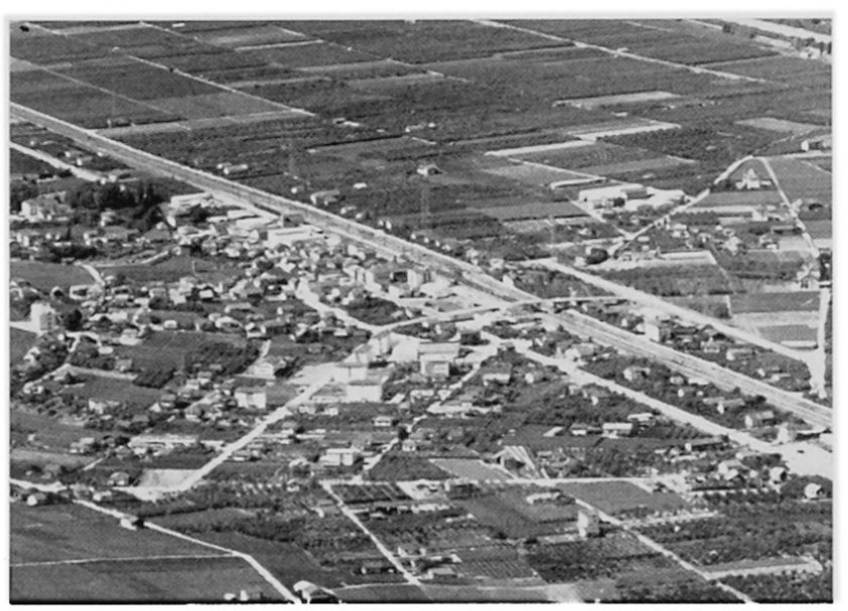


obstacles, mais il n'accuse qu'une légère accélération de courant, la dénivellation entre les deux compartiments de vallée étant très modeste.

A la hauteur de St-Pierre-de-Clages, une sorte de cône superposé s'individualise à la faveur d'un talus, par endroits très accentué; on l'attribue à un important débordement de la Losentze, consécutif à des glissements de terrain et à des pluies diluviennes de la fin du XIVème siecle. Cette inondation aurait entre autre contribué à l'enlisement partiel de la vieille église romane. Les gros blocs - en partie ensevelis - perdus au milieu des vignes ainsi que les amas de cailloux supportant quelques vieux chemins seraient également des témoins de l'épierrage qui a suivi cette catastrophe. Au nord et au nord-est de Chamoson, on distingue encore les traces d'un petit ravin dans lequel sévissait, il y a un siècle à peine, le torrent Merdenson (aujourd'hui St-André), dévié dans le large et profond lit endigué de la Losentze.
L'énorme amas d'alluvions récentes accumulées par la Losentze bouscule le Rhône contre les falaises de la rive gauche. Cette action devait etre plus efficace autrefois puisque, face à St-Pierre-de-Clages, toute une partie de l'ancienne falaise s'est précipitée dans la vallée, batissant le talus d'éboulis d'Arbin que des ruisseaux, nés du glissement, ont tapissé de matériel plus fin. Les limites de la niche d'arrachement entaillent nettement le versant entre Fey et Isérables.

La zone de contact entre le coteau sud et la plaine est abrupte de Riddes à Ecône. Plus à l'ouest, trois cônes de déjection consécutifs ont de tout temps offert aux hommes des terres alluviales cultivables, seuls terroirs figurant dans l'atlas Siegfried de 1885 .

\section{Le paysage végétal de la plaine}

Il n'est plus guère possible de déterminer ce que fut, il y a quelques lustres, la végétation naturelle de la plaine qui s'étend de Riddes a Martigny. Les roselieres bordant les rares étangs ou les canaux, les "rideaux-abris" de

Fig. 4: Cône d'éboulis d'Arbin et niche d'arrachement

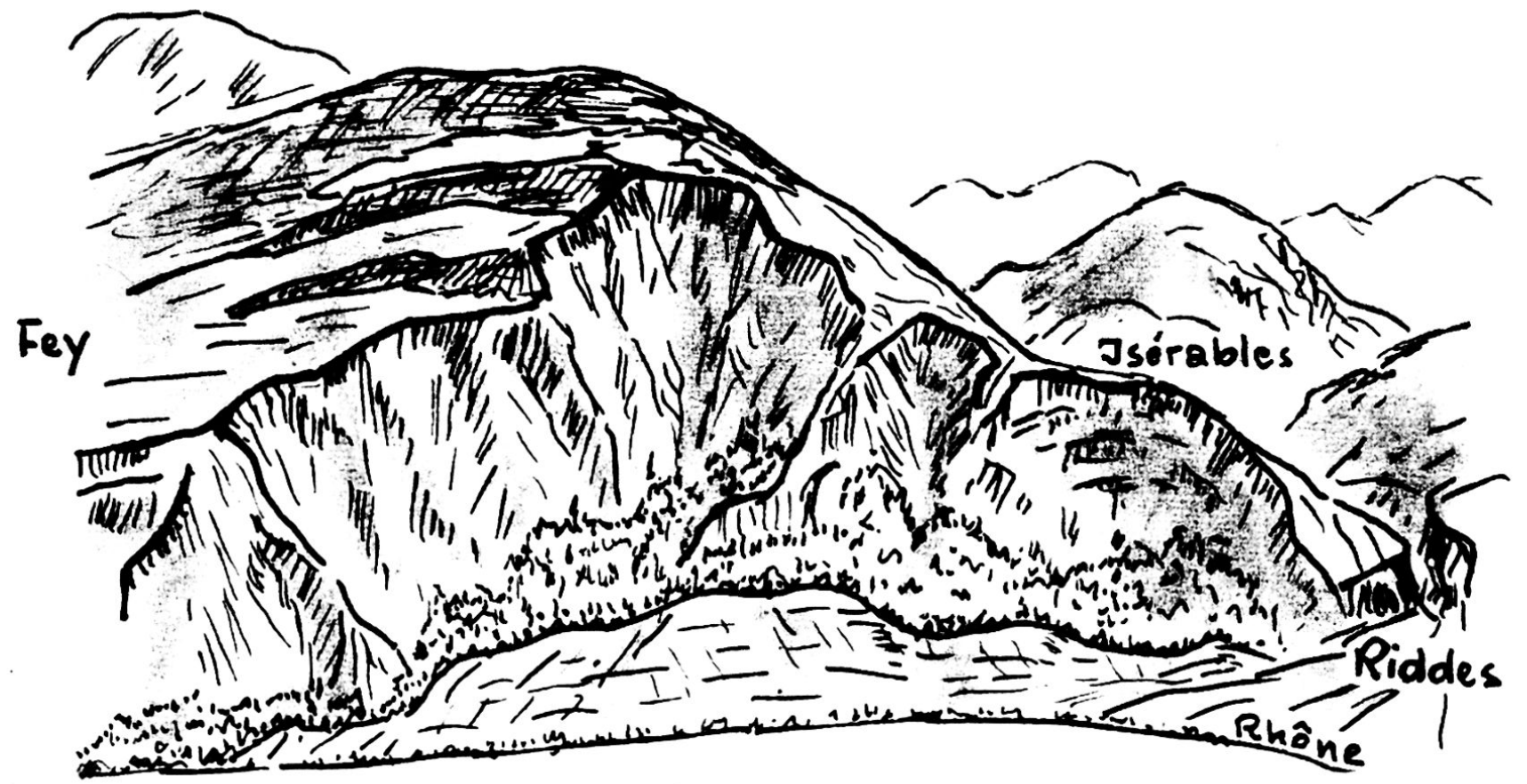


Saillon ainsi que la rangée de peupliers longeant le lit de la Drance en aval de Martigny en sont les seuls témoins. Une enqête menée auprès de vieilles gens, la consultation de photos ou de cartes topographiques, la visite des réserves naturelles de Grône ou de Sion permettent d'affirmer que tout le reste résulte du travail persévérant de l'homme.

Le jardin d'abricotiers occupe la plus grande partie des territoires de Saxon et de Charrat; les pommiers, les poiriers et les cultures marafcheres sont la ressource principale de la plaine de Fully et Saillon. L'éventail de la production agricole de Riddes est un résumé de ce que l'on trouve dans les diverses autres communes. Entre Martigny et Saxon, la plupart des arbres de haute futaie sont directement influencés par la brise du lac qui remonte régulièrement vers le Valais central, en zigzaguant d'un versant à l'autre; leurs branches s'orientent en fonction de ce vent dominant.

Ici et là, quelques hectares de vigne - plantés dans les graviers de cette zone interdite qu'est la plaine - peuvent surprendre:il s'agit de cultures antérieures à l'établissement du cadastre viticole et qui jouissent de ce fait d'une faveur spéciale. Les alluvions de la Salentze et de la Losentze qui portaient autrefois (atlas Siegfried 1915) de nombreuses prairies et vergers se sont habillées depuis quelques années de magnifique vignobles. La seule région encore en prairies s'étend en aval de Martigny, le long de la Drance. Cet espace est quadrillé par des bisses d'irrigation que l'alluvionnement et le curage réguliers ont surélevés.

L'évacuation des excédents d'eau de la plaine marécageuse a nécessité la construction d'un réseau de dra mage très dense. Il se hiérarchise pour aboutir finalement a deux canaux principaux qui côtoient le Rhône de part et d'autre. L'écoulement des eaux, premier souci des paysans d'autrefois, passe aujourd'hui au second plan puisque les "polders" du Valais doivent actuellement être irrigués. En effet, l'assechement des sols s'accentue d'année en année; la nappe phréatique est parfois si basse que les arbres même pâtissent de la sécheresse. Le climat estival de la région étant presque aussi aride que celui du Valais central, un apport artificiel d'eau se révele de plus en plus nécessaire. On le réalise en élevant le niveau des eaux souterraines à l'aide d'écluses qui entravent le libre écoulement dans les canaux de drainage. Là où cette méthode est inefficace, on pompe l'eau de la nappe pour la distribuer par aspersion. Ce dernier systeme exige des installations couteuses, mais il offre l'avantage d'etre utilisable également pour la protection contre le gel.

4. Le coteau de la rive droite

Aspects géologiques et morphologiques. Ce

Moyennes mensuelles et annuelles des précipitations de quelques stations de la vallée du Rhône

$\begin{array}{lllllllllllllll} & \text { Altit. } & \text { J } & \text { F } & \text { M } & \text { A } & \text { M } & \text { J } & \text { J } & \text { A } & \text { S } & \text { O } & \text { N } & \text { D } & \text { Total } \\ \text { Chessel } & 380 \mathrm{~m} . & 67 & 57 & 82 & 84 & 88 & 119 & 121 & 123 & 100 & 102 & 83 & 89 & 1115 \\ \text { Aigle } & 420 \mathrm{~m} . & 58 & 50 & 72 & 74 & 75 & 98 & 107 & 107 & 85 & 85 & 65 & 77 & 953 \\ \text { Bex } & 427 \mathrm{~m} . & 61 & 55 & 76 & 75 & 73 & 99 & 114 & 111 & 84 & 82 & 66 & 79 & 975 \\ \text { Lavey } & 440 \mathrm{~m} . & 63 & 54 & 83 & 88 & 82 & 98 & 113 & 115 & 92 & 90 & 75 & 80 & 1033 \\ \text { Martigny } & 471 \mathrm{~m} . & 63 & 52 & 62 & 61 & 49 & 59 & 72 & 75 & 63 & 71 & 61 & 83 & 771 \\ \text { Riddes } & 491 \mathrm{~m} . & 44 & 40 & 47 & 40 & 36 & 44 & 51 & 57 & 47 & 54 & 49 & 62 & 571 \\ \text { Sion(plaine) } & 483 \mathrm{~m} . & 47 & 40 & 44 & 40 & 38 & 44 & 49 & 58 & 46 & 54 & 49 & 63 & 572\end{array}$


coteau présente deux visages très différents. La région qui va des Follatères à Saillon est en pente tres raide; les terrasses sont absentes. Les empreintes de l'érosion vont dans le sens de la plus grande pente. Des ravins parfois profonds le coupent de haut en bas et animent un peu les pentes dominant Fully. A l'est de Mazembroz, la massivité du coteau s'accentue; il plonge vers la plaine en falaises imposantes que deux ou trois glissements de terrain (Chamouaire, Cleusette) - bien visibles de notre belvédère - réussissent à peine à entamer. Cette partie du versant droit de la vallée se caractérise en outre par une teinte brune généralisée a toutes les surfaces dépourvues de forêts. En sa partie supérieure et orientale, le changement est complet:des bancs de rochers plus clairs, disposés horizontalement, se superposent pour dessiner le Grand-Chavalard puis la Grande Garde. Ces couches plongent vers la plaine à la hauteur de Saillon, décrivant un vaste arc de cercle.

Nous voyons ici la zone de contact entre le massif cristallin des Aiguilles Rouges, qui constitue la partie inférieure du coteau et la nappe de Morcles, la plus profonde des $\mathrm{Hel}$ vétides. De cette dernière, il ne reste, à la hauteur de Saillon, que la flanc renversé ainsi que la partie centrale. Plus loin, au-dela de Chamoson, le flanc supérieur déploie d'amples falaises dans lesquelles nous pouvons suivre les ondulations de la nappe. Dans la zone de contact, au-dessus du hameau de la Sarvaz, du marbre est exploité jusque tres haut dans la montagne. D'autre part, au niveau de la plaine, entre le cristallin et le sédimentaire, jaillit une des plus abondantes sources de la vallée du Rhône valaisan; endiguée dès le début des travaux d'assainissement, elle s'écoule par les canaux de la Sarvaz et de Fully, jusqu'au-delà des Follatères où elle rejoint le fleuve.

Au-dessus de Saillon, la topographie du coteau est très étroitement liée à la succession et a la nature des couches géologiques partiellement arrachées. L'érosion glaciaire y a travaillé de façon différenciée, dessinant de petits replats séparés par des crêtes rocheuses.

La vaste échancrure en fer à cheval d'Ovronnaz

Fig. 5: La zone des racines de la nappe de Morcles

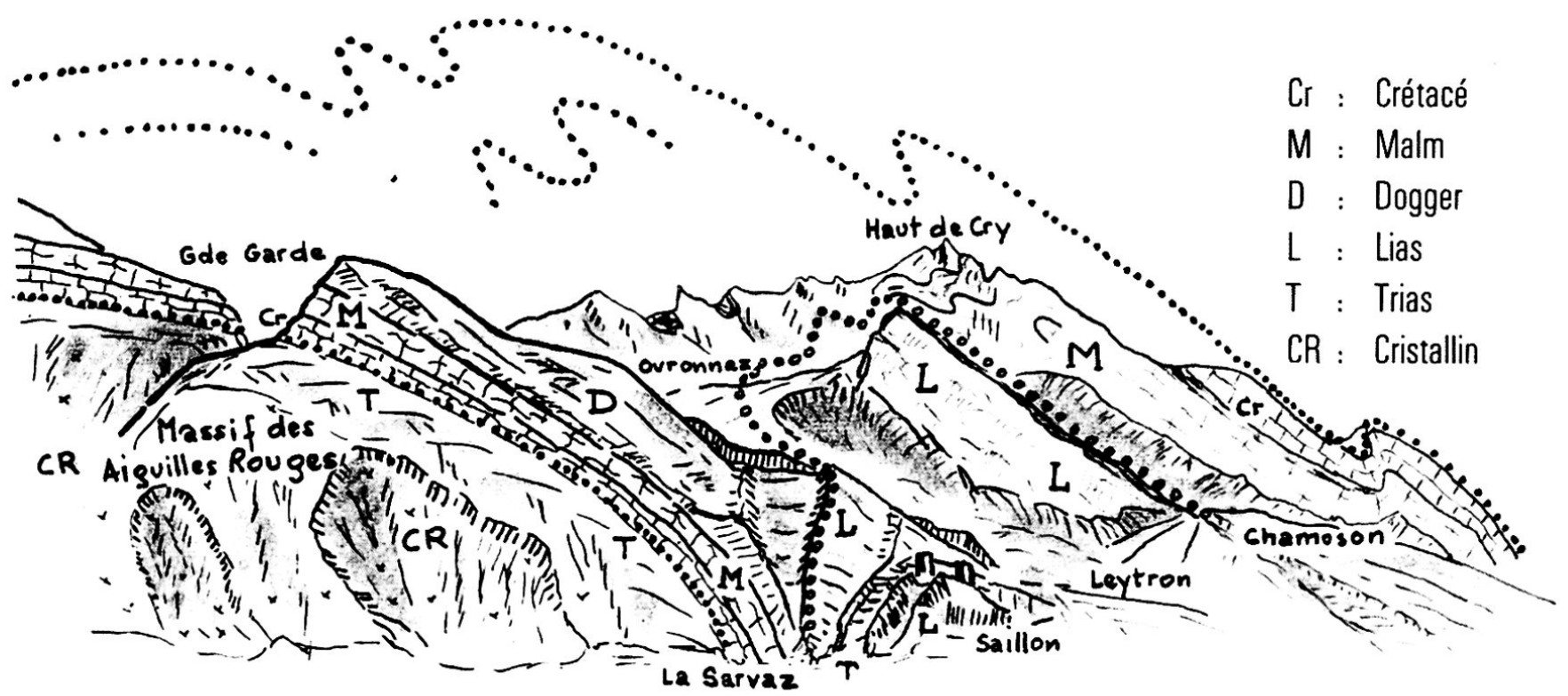


Fig. 6: Le coteau fluent au-dessus de Leytron (d'après Guide Géologique de la Suisse)

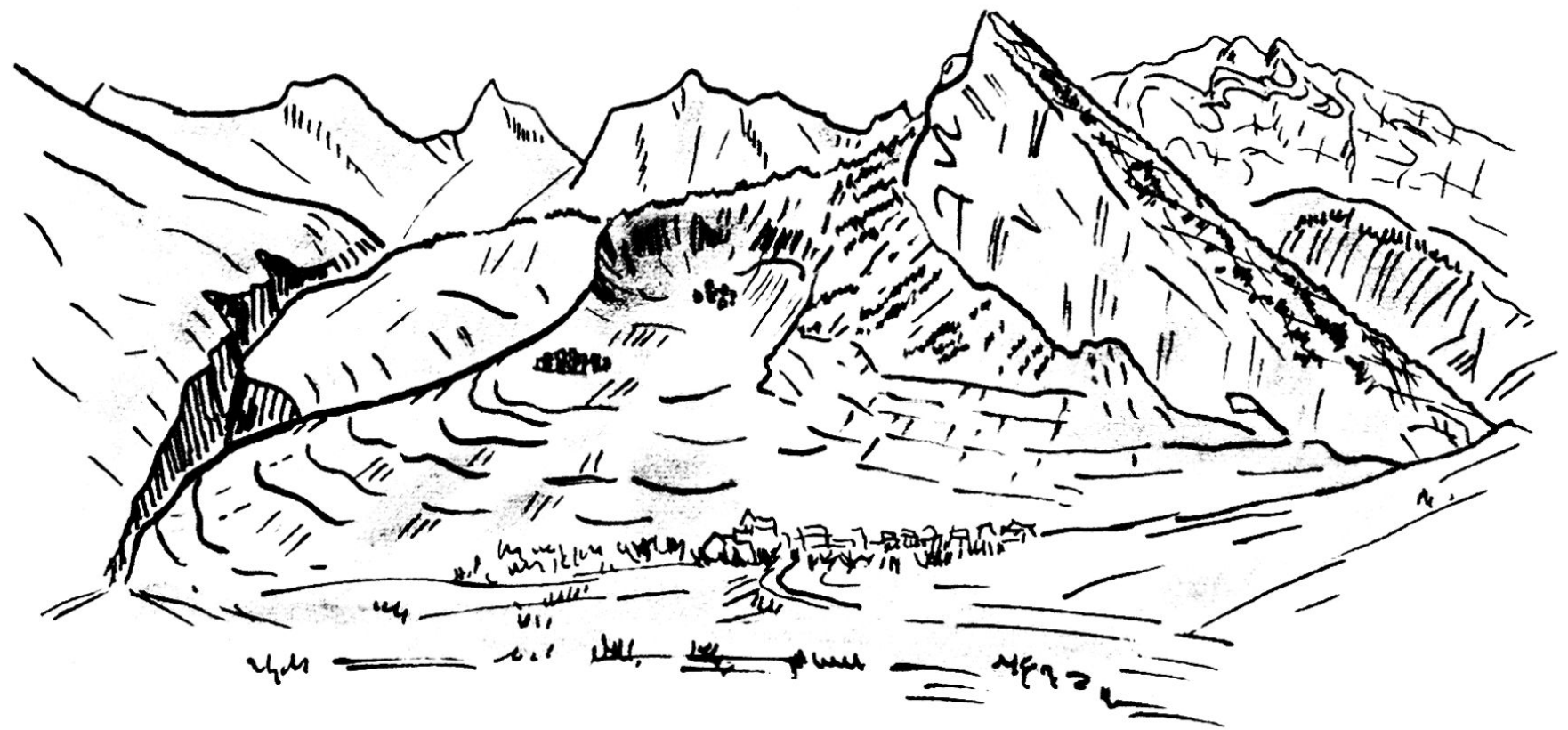

est taillée dans une épaisse couche de schistes tendres (Aalénien). L'éperon de l'Ardeivaz et la colline de Saillon sont des saillants liasiques plus durs situés au coeur de la nappe de Morcles.

Entre la Salentze - qui débouche sur la plaine par une profonde et étroite gorge - et Leytron, l'ensemble du coteau nord est en mouvement. Une niche d'arrachement se dessine à la hauteur d'Ovronnaz et de Dugny, cependant que s'édifie progressivement, en contrebas, une énorme loupe de glissement. Nichés au milieu des vignes, les villages de Produit et Monta gnon bravent l'instabilité du coteau.

Le vallon de la Losentze cache également de nombreux glissements de terrain; mais ici un torrent aux eaux souvent abondantes a déblayé ré gulièr ement le matériel qui obstruait son lit et l'a abandonné dans la plaine où il a édifié le cône de Chamoson. Cette évacuation continue a en outre activé les processus d'érosions et contribué au creusement du haut vallon de la Losentze. Il est possible aussi que quelques tassements ou éboulements de matériel plus massif descendu du Haut de Cry ou de l'Ardeivaz aient, à un stade initial, favorisé l'édification de l'énorme cône de déjection en lui donnant une assise peu mobile a la hauteur de Chamoson. Les nombreux rochers isolés que l'on trouve dans la région des cretes, à l'est $\mathrm{du}$ village, permettent cette hypothèse. Audessus de Leytron, l'absence de rivière importante a, au contraire, permis la subsistance du plateau d'Ovronnaz, malgré la fragilité de la roche.

\section{Le paysage végétal du coteau nord}

La végétation naturelle est très particuliere dans la première des régions. Les seuls arbres de haute futaie du bas coteau:hetres, chenes, ou chataigniers croissent sur la partie amont, non défrichée, des cônes de Fully. Audessus de Vers-l'Eglise, une chataigneraie aux arbres plusieurs fois centenaires était, avant la mise en valeur de la plaine, l'objet de soins attentifs; elle n'est plus aujourd'hui qu'un lieu de promenade:l'irrigation étant abandonnée, les fruits en souffrent et restent très petits.

Sur le reste du coteau, dans la foret, croissent surtout des feuillus de taille modeste auxquels se mêlent par-ci par-là quelques pins sylvestres. Les rochers dominant Branson ou ceux qui tombent en falaises à l'est de $\mathrm{Ma}$ - 
zembroz ne portent que des buissons ou des herbes seches. Sur les surfaces moins abruptes, tapissées de matériel triasique et d'éboulis calcaires pousse la forêt d'épicéas; elle devient plus dense avec l'altitude.

Malgré son accès difficile, l'étage inférieur du versant est très activement défriché pour être livré à la vigne. Cette culture chasse également les buissons dans les éboulis sis à l'est de Mazembroz.

Sur les racines de la nappe de Morcles et jusq'à près de 800 metres, la vigne remplace également les broussailles là où le rocher n'est pas à nu. Plus haut, dans le domaine de l'épicéa et du pin, le reboisement naturel rétrécit d'année en année les rares paturages des "mayens".

Les pentes mouvantes dominant Leytron sont, elles aussi, réservées à la vigne; elle monte ici progressivement vers le haut du coteau, presque sans murs de soutènement. L'instabilité du sol a sans doute contribué à l'abandon des murets de pierres sèches. Au-dessus du périmetre viticole (cote 800 ), les prairies, les vergers et les taillis occupent un territoire qui retient de moins en moins l'attention des agriculteurs.

6. Le coteau sud de la vallée du Rhône

Aspects géologiques et morphologiques. Ce coteau est d'abord abrupt entre Martigny et Charrat,malgré la série de petits cônes qui festonnent le pied des versants. Le massif du Mont Blanc, symétrique de celui des Aiguilles Rouges, dessine la croupe du Mont Chemin puis s'incline vers la plaine a la hauteur de Saxon. Les traces de l'érosion $s^{\prime}$ orientent selon la plus grande pente; elles sont bien apparentes, malgré l'épais manteau végétal. A partir de Charrat,le noyau cristallin est coiffé à son étage supérieur par des couches sédimentaires appartenant au flanc renversé de la nappe du Grand-St-Bernard. Les falaises dessinées par cette couverture sont moins individualisées que celles de la rive droite. Leur teinte plus claire permet de les suivre dès le col des Planches. Elles dessinent de vastes arcs de cercle qui plon- gent vers la plaine à la hauteur de Saxon. Elles adoucissent la pente sud entre Charrat et Ecône et cachent, grâce à leur lithologie variée, de très nombreux replats tapissés de moraines quaternaires.

Au point de contact entre le massif du Mont Blanc et la zone de Ferret jaillit - a plus de $25^{\circ} \mathrm{C}-1$ 'abondante source thermale de Saxon, autrefois activement exploitée et aujourd'hui en quete d'investisseurs. Aux environs de Riddes, le versant s'élève, très abrupt, jusqu'à plus de 1000 metres.

7. Le paysage végétal du coteau sud

La forêt de hêtres prospère à l'ouest de Charrat, sur les flancs du Mont Chemin; la brise du lac que la Cluse de St-Maurice dirige vers le coteau sud vaut à la région une humidité relative nettement supérieure à celle du Valais central. Plus haut, sur les sols morainiques du col des Planches et du col du Lin, le mélèze remplace les feuillus. Dès Saxon, le hêtre disparaft; il est absent de la forêt naturelle dominant Ecône ou Riddes. Etait-ce aussi le cas au-dessus de Saxon? Le défrichement généralisé n'a épargné que les surfaces impropres a la culture; il n'est donc plus possible, par l'observation directe, de tracer la limite végétale déterminée par l'advection régulière d'air lémanique; seuls quelques individus isolés se perdent au milieu des épicéas, des pins et des mélèzes le long du torrent des Croix. De Charrat a Ecône, les surfaces exploitées du coteau sont réservées à l'abricotier, qui prospère jusqu'à plus de 1000 metres aux environs de Sapinhaut. Le verger cède cependant le pas au vignoble, aux alentours immédiats de Charrat et de Saxon.

\section{BIBLIOGRAPHIE}

\section{A) Cartes}

Atlas Siegfried, éch. 1:50'000, F. No 485 et 52 . (1880 a 1932)

Carte nationale de la Suisse, éch. 1:25'000, No 1305 et 1325

B) Géologie et morphologie Gerber E.K.: Morphologische Untersuchungen im Rhonetal. Schinznach. Lerchmüller- 
Müri, 1944.

Hartmann P. : Zur Geologie des Kristallinen Substratums der Dent-de-Morcles. Luzern. Wigger 1915. Th. sc. Fribourg. Horwitz L. : Contribution à l'étude des cônes de déjection dans la vallée du Rhône (bull. Sté vaudoise des sc. nat. , vol. 47, No 173) Lausanne, Th. sc.

Lugeon M. : Sur l'ampleur de la Nappe de Morcles (Paris 1914)

Le glissement des hameaux de Montagnon et Produit. Comm. de Leytron. St-Maurice 1932.

Schardt H. : L'éboulement du Grugnay près de Chamoson. Bull. de la Murithienne du Valais. T. 341907.

C) Aspects humains et économiques Giroud Edm. : Chamoson, aspect et proble - mes d'une commune paysanne et vigneronne. Sion, Gessler, 1964.

Loup Jean: Pasteurs et agriculteurs valaisans. Grenoble:Allier, 1965, Th. L.

D) Climat

Roten M.: Recherches microclimatiques sur la vallée du Rhône en Valais. Th. phil. Fribourg, 1964 .

E) Végétation

Frey H. : Die walliser Felsensteppe. Diss. phil. II, Zürich, 1934.

Hainard P. : Signification écologique et biogéographique de la répartition des essences forestieres sur l'adret valaisan. Th. sc. Geneve.

\section{Literaturbesprechung}

GERSTER G. : Der Mensch auf seiner Erde, eine Befragung in Flugbildern; 31l S. , wovon 192 Bild-S., 96 S. farbig. Verlag Atlantis, Zürich/ Freiburg i. B., 1975. DM/sFr. 115. 00.

Mit diesem grossformatigen Flugbildwerk wird man mit einer Dokumentations-Sammlung beschenkt und vertraut gemacht, die in Form und Inhalt von keinem andern Band übertroffen werden kann. Allein die Tatsache, dass Gerster kaum ein Thema beiseite lässt, das zwischen Mensch und Erde von Belang ist, muss auf uns Geographen sehr attraktiv wirken:Diese Grundmuster unseres menschlichen Daseins sind das Ergebnis einer zehnjährigen photographischen Detailarbeit.

In dieser "aus der Luft begriffenen"Schau, wird bewusst jeder Mensch zur Verantwortung gezogen, der in die Naturabläufe eingreift oder sich ihnen in dieser oder jener Form anpassen will oder muss. Auch der Nicht-Geograph schätzt ganz besonders, dass die brillanten Aufnahmen aus fünfzig Ländern nach thematischen Kategorien wohlgeordnet sind. -Ein Grosskapitel ist dem Bauern, dem wichtigsten Kulturlands chafts- gestalter, gewidmet. Gerster sieht auch den modernen Bauern und weist ihn bewusst auf erosionsgerechte Anbaumethoden. Viele Flugbilder greifen in die Vergangenheit zurück, in biblische Städte mit Kulturzentren oder in Siedlungsformen des industriell modern wirtschaftlichen Menschen. Gerster's Dokumentation entbehrt das Spiel mit optischen Effekten; er schätzt die sehr nüchtern präsentierte Realität und wirkt dadurch wahrhaftiger als eine blosse Photoschau. Für den Geographielehrer ist zu bedauern, dass er für seinen anschaulich sein sollenden Unterricht nur einen festgebundenen Bildband erstehen kann. Er würde sich eine Samm lung von gelochten Einzelblättern wünschen, möglicherweise auch als Einzeldiapositive, um dem Schüler "den Menschen auf seiner Erde" audio-visuell noch leichter als bislang näher bringen zu können. Vielleicht übernimmt die "Swissair", die von Anfang an dem wahrlich einmaligen Werk grosse Impulse verlieh, den Gedanken auf, auch in dieser pädagogischen Richtung mitzuwirken.

Werner Kündig-Steiner 